\title{
THE NICHOLSON-VARADARAJAN THEOREM ON CLEAN LINEAR TRANSFORMATIONS
}

\author{
VICTOR CAMILLO \\ Department of Mathematics, The University of Iowa, Iowa City, IA 52242 U.S.A. \\ e-mail: camillo@math.uiowa.edu \\ and JUAN JACOBO SIMÓN \\ Departamento de Matematicas, Universidad de Murcia Spain, 30100 Murcia, Spain \\ e-mail: jsimon@fcu.um.es
}

(Received 31 October, 2000; accepted 30 March, 2001)

\begin{abstract}
An element $r$ in a ring $R$ is clean if $r$ is a sum of a unit and an idempotent. Camillo and $\mathrm{Yu}$ showed that unit regular rings are clean and in a very surprising development Nicholson and Varadarajan showed that linear transformations on countable dimension vector spaces over division rings are clean. These rings are very far from being unit regular.

Here we note that an idempotent is just a root of $g(x)=x^{2}-x$. For any $g(x)$ we say $R$ is $g(x)$-clean if every $r$ in $R$ is a sum of a root of $g(x)$ and a unit. We show that if $V$ is a countable dimensional vector space and over a division ring $D$ and $g(x)$ is any polynomial with coefficients in $K=\operatorname{Center} D$ and two distinct roots in $K$, then End $V_{D}$ is $g(x)$-clean.
\end{abstract}

2000 Mathematics Subject Classification. Primary 16S50, Secondary 16E50.

1. Introduction. A ring $R$ is clean if every $r \in R$ is the sum of a unit and an idempotent. In [1] it was shown that a unit regular ring is clean. Now a regular ring is unit regular if and only if $f R \approx g R$ implies $(1-f) R \approx(1-g) R$. Therefore it was quite surprising when Nicholson and Varadarajan showed that every linear transformation on a space of countable dimension over a division ring is clean.

Think of an idempotent as a solution to $g(x)=x(x-1)=0$. We seek to replace $x(x-1)$ by a more general $g(x)$. Suppose $D$ contains a field $K$ and $g(x) \in K[x]$. Consider the one-dimensional case and $d \in D$, then, $d=s+u$ where $g(s)=0$ and $u$ is a unit. Since $u$ is a unit, $u \neq 0$. Let $s=t+v$ where $g(t)=0$. Since $v \neq 0$, we must have that $g$ has at least two roots in $D$.

Let $R$ be a ring that contains a field $K$. Let $g(x) \in K[X]$. We say that $R$ is $g(x)$ clean if for every $r \in R$ we have $r=s+u$ where $g(s)=0$ and $u$ is a unit. We have seen from the above paragraph that if $R$ is $g(x)$-clean, then $g(x)$ has at least two roots in $R$. However, even if $R$ is a division ring, we cannot factor $g(x)$ because of commutativity problems. Therefore, we assume $g(x)$ has two roots in $K=\operatorname{Center} D$. In the Nicholson-Varadarajan Theorem, these are 0 and 1.

This paper was written while the first author was visiting the University of Murcia under a grant from The Foundation Séneca. CARM. Supported by DGES (PB96-0961-C02-02) and CARM (PB/16/FS/97). 
We prove here:

THEOREM 1.1. Let $V_{D}$ be a vector space of countable dimension or less over a division ring $D$. Let $g(x)$ be a polynomial with at least two roots in $K=\operatorname{Center} D$. Then End $V_{D}$ is $g(x)$-clean.

COROLlary 1.2. If $D$ is commutative then $V$ is $g(x)$-clean for all spaces of countable dimension if and only if $g(x)$ has at least two roots in $K$.

In what follows we number our lemmas as in [3].

2. The Shift Operator. Let $V_{D}$ be a vector space of countable dimension with basis $\left\{x_{i} \mid i \in N\right\}=X$. Let $s\left(x_{i}\right)=x_{i+1}$ be the associated shift operator. Let $g(x)=(x-u)(x-v)$ be a polynomial of degree two that splits as shown. Let $L \in$ End $V$. We want to show eventually that $L=a+u$ where $g(a)=0$ and $u$ is a unit. Nicholson and Varadarajan have shown that if $g(x)=x^{2}-x$ it is enough to do this for $L=s$, the shift operator $s$ with respect to the basis $X$. To do this we must find an $a$ with $g(a)=0$ and $a-s$ a unit. First we have:

$$
(x-u)(x-v)=(x-u)(x-u+u-v)=0 .
$$

So

$$
(x-u)^{2}=(u-u)(x-u) .
$$

Let $l=(u-v)$.

We illustrate our techniques with some preliminary considerations.

It is clearly enough to define $a-s$. We begin with:

$$
(a-s) x_{1}=p x_{1} .
$$

Then ask what $(a-s) x_{2}$ must be for $(a-u)^{2}=l(a-u)$.

We have $a x_{1}=p x_{1}+x_{2}$ so $(a-u+u) x_{1}=p x_{1}+x_{2}$,

$$
(a-u) x_{1}=(p-u) x_{1}+x_{2} .
$$

We multiply both sides of $(2.1)$ by $(a-u)$ to obtain

$$
(a-u)^{2} x_{1}=(p-u)(a-u) x_{1}+(a-u) x_{2} .
$$

Now, we want $(a-u)^{2} x_{1}=l(a-u) x_{1}$, that is,

$$
\begin{aligned}
l(a-u) x_{1} & =(p-u)(a-u) x_{1}+(a-u) x_{2}, \\
(l-p+u)(a-u) x_{1} & =(a-u) x_{2}, \\
(l-p+u)(a-u) x_{1} & =(a-s+s-u) x_{2}, \\
(l-p+u)(a-u) x_{1} & =(a-s) x_{2}+x_{3}-u x_{2}, \\
(l-p+u)(a-u) x_{1}+u x_{2}-x_{3} & =(a-s) x_{2} .
\end{aligned}
$$

Use the fact that $a x_{1}=p x_{1}+x_{2}$ so $(l-p+u)\left(p x_{1}+x_{2}-u x_{1}\right)+u x_{2}-x_{3}=(a-s) x_{2}$. Now collect terms: 


$$
(l-p+u)(p-u) x_{1}+(l-p+2 u) x_{2}-x_{3}=(a-s) x_{2} .
$$

Now, the right side of (2.3) can be obtained by reversing the steps to (2.4) and is also the right side of (2.2). This means that (2.4) is necessary and sufficient for $(a-u)^{2} x_{1}=l(a-u) x_{1}$. This suggests a guess for the general case.

Proposition 2.1. Let $V_{D}$ be a vector space of countable dimension over a division ring $D$ containing a field $K$. Let $g(x)=(x-u)^{2}-l(x-u)$ where $u$ and $l$ are in $K$. Let $X_{0}=\left\{x_{i} \mid i \in N\right\}$ be a basis for $V$ with shift operator $s$. In $A$ and $B$ below if $p \neq 0$ and $l+p+2 u \neq 0$ then $A$ and $B$ define $a$ so that $a-s$ is $a$ unit and $g(a)=0$.

A. $(a-s) x_{2 k-1}=p x_{2 k-1}$.

B. $(a-s) x_{2 k}=(l-p+u)(p-u) x_{2 k-1}+(l+p+2 u) x_{2 k}-x_{2 k+1}$.

Proof. We factor B to obtain

$$
(a-s) x_{2 k}=(l-p+u)\left[p x_{2 k-1}-u x_{2 k-1}+u x_{2 k}\right]+u x_{2 k}-x_{2 k+1} ;
$$

then from A,

$$
p x_{2 k-1}-u x_{2 k-1}+u x_{2 k}=(a-u) x_{2 k-1} .
$$

So

$$
(a-s) x_{2 k}=(l-p+u)(a-u) x_{2 k-1}+u x_{2 k}-x_{2 k+1}
$$

then cancel $-s x_{2 k}=-x_{2 k+1}$, bring $u x_{2 k}$ to the left side of (2.8) and obtain

$$
\begin{aligned}
(a-u) x_{2 k} & =(l-p+u)(a-u) x_{2 k-1}, \\
(a-u) x_{2 k-1} & =p x_{2 k-1}-u x_{2 k-1}+x_{2 k} .
\end{aligned}
$$

So, from A, multiplying the above by $(a-u)$ and factoring:

$$
(a-u)^{2} x_{2 k-1}=(p-u)(a-u) x_{2 k-1}+(a-u) x_{2 k} .
$$

Substitute from (2.9) for $(a-u) x_{2 k}$ and cancel. We get

$$
(a-u)^{2} x_{2 k-1}=l(a-u) x_{2 k-1} .
$$

Further, from (2.9), $(a-u) x_{2 k}$ is a scalar multiple of $(a-u) x_{2 k-1}$ so $(a-u)^{2} x_{2 k}=$ $l(a-u) x_{2 k}$ also, which establishes that $a$ satisfies $p(x)$.

To show that $(a-s)$ is a unit, first note it is onto because by $\mathrm{A}, \operatorname{Im}(a-s)$ contains all the basis vectors indexed by odd natural numbers and therefore by $\mathrm{B}$ the even index ones also, since we are assuming $p \neq 0$ and $l+p+2 u \neq 0$.

To see that $(a-s)$ is one to one, suppose $\sum \lambda_{i}(a-s) x_{i}=0$. Let $\lambda_{i_{0}}$ be the largest nonzero coefficient. Suppose $i_{0}$ is even, $i_{0}=2 j_{0}$. Then when we expand the relation in terms of the basis $X,-\lambda_{i_{0}}$ appears as the coefficient of $x_{2 j_{0}+1}$ exactly once and so is zero.

If $i_{0}=2 j_{0}-1$ is odd, then $\lambda_{2 j_{0}-1}\left(p x_{2 j_{0}-1}\right)+\lambda_{2 j_{0}-2}\left[(l-p+u)(p-u) x_{2 j_{0}-3}+\right.$ $\left.(l+p+2 u) x_{2 j_{0}-2}>+x_{2 j_{0}-1}\right]+\cdots=0$. Then, checking the coefficient of $x_{2 j_{0}-2}$ we have $\lambda_{2 j_{0}-2}=0$ and we are reduced to the previous case. 
3. The general case. Nicholson and Varadarajan show how to extend the truth of their theorem in the shift operator context to the entire space under consideration. Their argument works here. We have to show their proof works. We repeat much of their argument as quickly as possible.

Lemma 3.1 If $R$ is $g(x)$-clean then any $n \times n$ companion matrix over $R$ is $g(x)$ clean.

Proof. Let $a_{1}=s+u$ where $g(s)=0$; then

$$
\begin{aligned}
{\left[\begin{array}{cccccc}
0 & 0 & 0 & \cdots & 0 & a_{1} \\
1 & 0 & 0 & \cdots & 0 & a_{2} \\
0 & 1 & 0 & \cdots & 0 & a_{3} \\
\vdots & \vdots & \ddots & \ddots & \vdots & \vdots \\
0 & 0 & 0 & \cdots & 0 & a_{n-1} \\
0 & 0 & 0 & \cdots & 1 & a_{n}
\end{array}\right]=} & {\left[\begin{array}{ccccc}
0 & 0 & 0 & \cdots & s \\
0 & 0 & 0 & \cdots & s \\
0 & 0 & 0 & \cdots & s \\
\vdots & \vdots & \vdots & & \vdots \\
0 & 0 & 0 & \cdots & s \\
0 & 0 & 0 & \cdots & s
\end{array}\right] } \\
+ & {\left[\begin{array}{cccccc}
0 & 0 & 0 & \cdots & 0 & u \\
1 & 0 & 0 & \cdots & 0 & a_{2}-s \\
0 & 1 & 0 & & 0 & a_{3}-s \\
\vdots & \vdots & \ddots & \ddots & \vdots & \vdots \\
0 & 0 & 0 & \cdots & 0 & a_{n-1}-s \\
0 & 0 & 0 & \cdots & 1 & a_{n}-s
\end{array}\right] . }
\end{aligned}
$$

The first matrix on the right side satisfies $g(x)=0$ and the second is a unit.

Lemma 3.2. Let a be $g(x)$-clean in the a-invariant subspace $U$. Suppose $V=$ $\operatorname{span}\left\langle a^{n}(y)\right\rangle+U$ for some vector $g$. Then a is $g(x)$-clean in $V$.

Proof. Let $V=M \oplus U$ where $g \in M$ and $y \in M$. Let $\mathcal{O}$ be the projection onto $M$ with kernel $U$. Let $\mathcal{O}_{0}: V / U \rightarrow M$ be the induced isomorphism, and $\bar{a}$ be the induced action of $a$ on $V / U$.

Then $\left\{\mathcal{O}_{0} \bar{a}^{n}(\bar{y})\right\}=X$ spans $M$. Proposition 2.1 applies if $X$ is a basis; Lemma 3.1 applies if $X$ is finite-dimensional. In either case $\mathcal{O}_{0} a \mathcal{O}_{0}^{-1}=a_{2}+b_{2}$ where $a_{2}: M \rightarrow M$ satisfies $g\left(a_{2}\right)=0$ and $b_{2}$ is an automorphism of $M$. By assumption we may write $a=a_{1}+b_{1}$ on $U$, where $g\left(a_{1}\right)=0$ and $b_{2}$ is a unit.

Let

$$
\begin{aligned}
& c(m+u)=a_{2}(m)+a_{1}(u), \\
& d(m+u)=b_{2}(m)+\left[a(m)-\mathcal{O}_{0} a \mathcal{O}_{0}^{-} 1(m)+b_{1}(U)\right] .
\end{aligned}
$$

Then, since $c$ is defined componentwise $g(c)=0$. Also

$$
\begin{aligned}
c(m+u)+d(m+u) & =\left[a_{2}(m)+b_{2}(m)-\mathcal{O}_{0} a \mathcal{O}_{0}^{-} 1(m)\right]+[a(m)]+\left[a_{1}(u)+b_{1}(u)\right] \\
& =0+a(m)+a(u) \\
& =a(m+u) .
\end{aligned}
$$


The reader may consult [3] to see that $d$ is a unit.

Now as in [3] given $a$, we create triples $\left(u, a_{i}, b_{i}\right)$ where $U_{1}$ is $a, a_{i}, b_{i}$ and $a=a_{i}+b_{i}$. These are partially ordered by containment and extension of maps. Pick a maximal triple and the previous lemmas show that $U=V$ and $a=a_{i_{0}}+b_{i_{0}}$.

\section{REFERENCES}

1. V. P. Camillo and H. P. Yu, Exchange rings, units and idempotents, Comm. Algebra 22 (1994), 4737-4749.

2. W. K. Nicholson, Lifting idempotents and exchange rings, Trans. Amer. Math. Soc. 229 (1977), 269-278.

3. W. K. Nicholson and K. Varadarajan, Countable linear transformations are clean, Proc. Amer. Math. Soc. 126 (1998), no. 1, 61-64. 